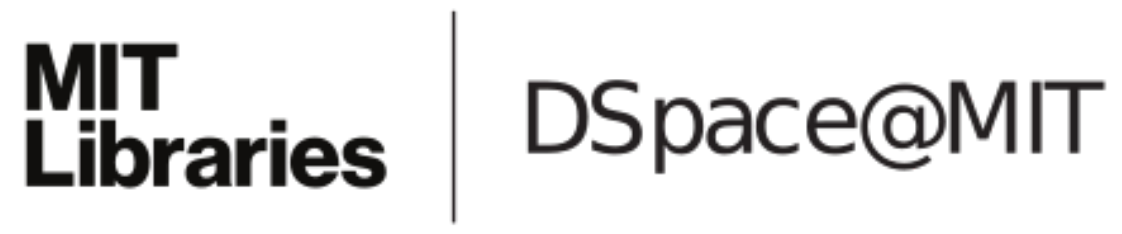

\author{
MIT Open Access Articles
}

On-wafer seamless integration of GaN and Si (100) electronics

The MIT Faculty has made this article openly available. Please share how this access benefits you. Your story matters.

Citation: Jin Wook Chung, Bin Lu, and T. Palacios. “On-Wafer Seamless Integration of GaN and Si (100) Electronics." Compound Semiconductor Integrated Circuit Symposium, 2009. CISC 2009. Annual IEEE. 2009. 1-4. (2009 Institute of Electrical and Electronics Engineers.

As Published: http://dx.doi.org/10.1109/csics.2009.5315780

Publisher: Institute of Electrical and Electronics Engineers

Persistent URL: http://hdl.handle.net/1721.1/53730

Version: Final published version: final published article, as it appeared in a journal, conference proceedings, or other formally published context

Terms of Use: Article is made available in accordance with the publisher's policy and may be subject to US copyright law. Please refer to the publisher's site for terms of use. 


\title{
On-Wafer Seamless Integration of GaN and Si (100) Electronics
}

\author{
Jin Wook Chung, Bin Lu and Tomás Palacios" \\ Department of Electrical Engineering and Computer Science and Microsystems Technology Laboratories \\ Massachusetts Institute of Technology, 77 Massachusetts Ave. Bldg. 39-567B, Cambridge, MA 02139, USA \\ "Corresponding author; phone: +1-617-324-2395; FAX:+1-617-258-7393; email: tpalacios@mit.edu
}

\begin{abstract}
The high thermal stability of nitride semiconductors allows for the on-wafer integration of (001) Si CMOS electronics and electronic devices based on these semiconductors. This paper describes the technology developed at MIT to seamlessly integrate $\mathrm{GaN}$ and Si transistors in very close proximity $(<5 \mu \mathrm{m})$. This integration, the first of any III-V field effect transistor with (001) $\mathrm{Si}$ electronics, enables tremendous new possibilities to circuit and system designers. For example, we will study the use of hybrid GaN-Si circuits to improve the power distribution networks in Si microprocessors.
\end{abstract}

Keywords: GaN, high electron mobility transistors, nitride electronics, high frequency, heterogeneous integration.

\section{INTRODUCTION}

Moore's law has been one of the main drivers behind the unprecedented development of semiconductors in the last forty years. However, this economical and technological paradigm that has helped to create modern $\mathrm{Si}$ electronics is now jeopardizing its future. Traditional Si scaling is not only becoming unaffordable, but the performance improvement due to scaling is diminishing.

Our group is working on an approach different from Moore's law to increase the performance of electronics: the heterogeneous integration of different semiconductor materials on the same wafer. In this paper, we describe our work on the seamless integration of $\mathrm{GaN}$-based devices and $\mathrm{Si}$ electronics. While Si electronics has shown unsurpassed levels of scaling and circuit complexity, nitride semiconductors offer excellent optoelectronics and high frequency/power electronic properties. The ability to combine these two material systems in the same chip and in extremely close proximity would allow unprecedented flexibility for advanced applications.

\section{HETEROGENEOUS INTEGRATION WITH SILICON DIGITAL ELECTRONICS}

The unique properties of $\mathrm{AlGaN} / \mathrm{GaN}$ High Electron Mobility Transistors (HEMTs) have made them the best option for many RF amplifiers. The unsurpassed high current levels possible in these devices [1], in combination with their very high breakdown voltage allow almost 10 times higher maximum power density than GaAs amplifiers [2]. In addition, their high frequency performance, an $f_{\max }$ of $300 \mathrm{GHz}$ has recently been demonstrated [3], enables extremely high gain and power added efficiencies. Also, the high output resistance resulting of the small device width significantly simplifies the design of the matching networks in RF amplifiers. Finally, the recent demonstration of device lifetimes in excess of $10^{6}$ hours at a channel temperature of $175 \mathrm{C}$ make this technology one of the most reliable semiconductor technologies [4]. 
In spite of the excellent performance demonstrated by nitride transistors, these devices cannot compete with Si MOSFETs in terms of scalability and level of integration. Modern microprocessors, for example, have more than one billion Si transistors on a single chip [5]. In spite of this unsurpassed scalability, traditional Si electronics is facing tremendous challenges to continue its scaling and performance improvement due to short channel effects and power dissipation. The onchip integration of nitride and $\mathrm{Si}$ technology would enable new flexibility in the circuit and device design to increase the system performance.

Previously, several authors have reported heterogeneous integration of $\mathrm{Si}$ and GaAs devices (i.e. field effect transistors, light emitting diodes), by the low-temperature selective epitaxial growth of GaAs on a miscut Si(100) substrate [6-8]. With similar technology, several groups have reported the growth of $\mathrm{GaN}$ structures on miscut $\mathrm{Si}(100)$ or $\mathrm{Si}(110)$ substrates by molecular beam epitaxy
(MBE) [9] and metalorganic vapor phase epitaxy (MOVPE) [10]. However, this approach is challenging because of the difficulty of growing high quality wurtzite $\mathrm{GaN}$ on (100)-oriented cubic Si substrates [10]. Moreover, the use of miscut substrates increases the density of surface states in the $\mathrm{Si}$ material, degrading the performance of $\mathrm{Si}$ electronics designed therein.

The technology to integrate GaN and $\mathrm{Si}$ electronics in the same wafer starts by fabricating a virtual Si (001) / GaN / Si (001) substrate by wafer bonding with a $\mathrm{SiO}_{2}$ bonding interlayer (Fig. 1) [11]. Due to the high thermal stability of GaN, Si CMOS electronics can then be processed in these new substrates without affecting the nitride layers underneath the surface. After the Si devices are fabricated, the Si material is removed from the regions where nitride devices are needed. Then, the nitride devices (transistors, LEDs, lasers or sensors) are processed and, finally, an interconnection layer forms the final hybrid circuits (Figs. 2 and 3).

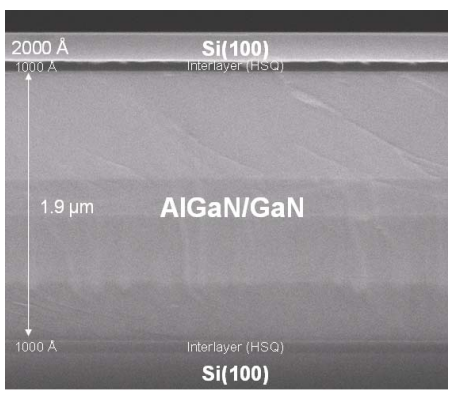

Fig. 1. Scanning electron micrograph of the cross-section of a Si/nitride/Si wafer.

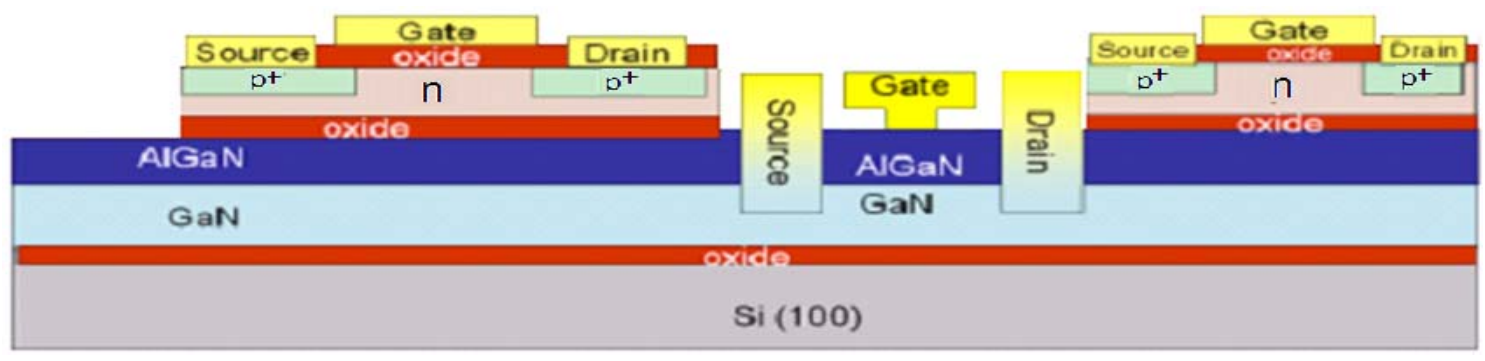

Fig. 2. Diagram showing the cross-section of a virtual wafer with Si pMOS transistors and Nitride HEMTs are fabricated in very close proximity. 


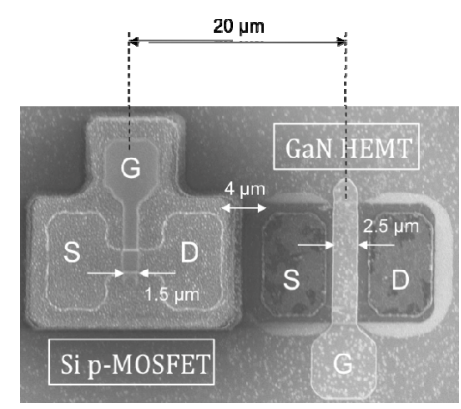

Fig. 3. Scanning electron micrograph of a GaN HEMT and a Si p-MOSFET fabricated side by side in a virtual wafer.

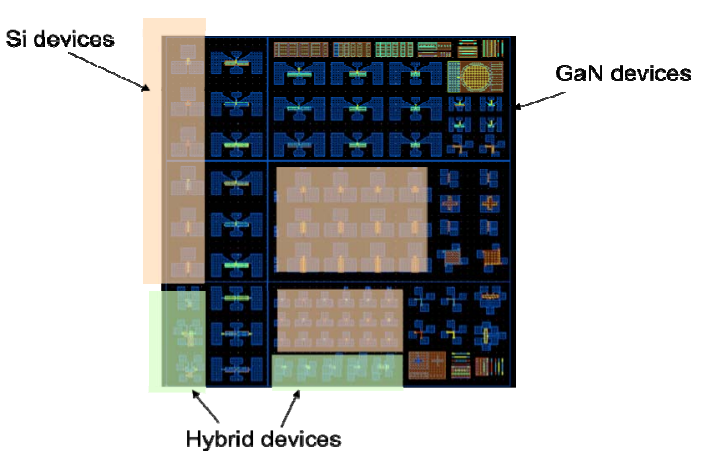

a)

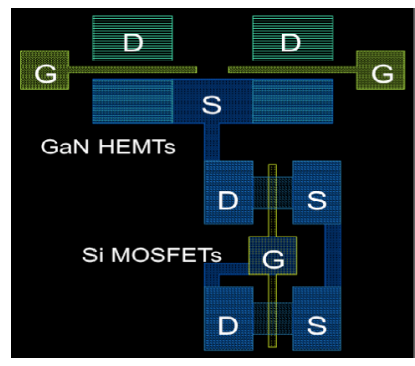

b)

Fig. 4. Diagram of the hybrid mask used in this work. Si devices and GaN devices are fabricated at the same time. b) shows the structure of a high power differential amplifier where the GaN devices form the differential pair and the Si MOSFETs act as a current source.

Using this new technology several the new design, although additional circuit hybrid circuits are currently being developed, including high power differential amplifiers, normally-off power transistors (Fig. 4) and highly compact DC-DC power converters for advanced power distribution in $\mathrm{Si}$ microprocessors.

As an example of the new hybrid GaN$\mathrm{Si}$ circuits currently under developing in our group, Fig. 5 shows the schematic of a GaN-Si hybrid power converter. In this hybrid circuit, switches M1 and M2 see the highest voltage stress and should be implemented with $\mathrm{GaN}$ transistors. In the other devices, the voltage stresses are much lower, which allow their implementation with $\mathrm{Si}$ electronics. This circuit is currently under fabrication and Fig. 6 shows the simulation results of the circuit described in Fig. 5. The operating frequency was $300 \mathrm{MHz}$ to minimize circuit area. Simulation verifies the excellent behavior of optimization is needed to improve the efficiency.

\section{CONCLUSIONS}

Nitride transistors have shown an outstanding improvement in performance during the last few years, which has allowed them to become the first option for power amplification below $10 \mathrm{GHz}$. However, the range of applications where nitride devices can revolutionize electronics does not stop in power amplifiers. Their high thermal stability allows their seamless integration with Si (100) devices, which enables numerous applications that take advantage of the unsurpassed integration density of $\mathrm{Si}$ electronics and the high breakdown and operating frequency of nitrides. This paper has demonstrated the first on-wafer integration of $\mathrm{GaN}$ and $\mathrm{Si}(100)$ devices and some of the new hybrid circuits allowed by this integration. 


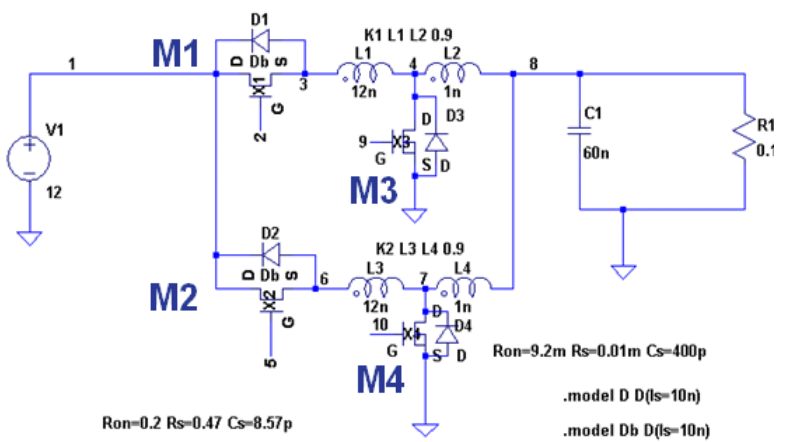

Fig. 5. Circuit schematic of the new GaN-Si hybrid power converter. M1 and M2 are GaNbased power transistors while M3 and M4 are Si MOSFETs.

\section{ACKNOWLEDGEMENTS}

The results described in this paper have been partially funded by the DARPA Young Investigator Award (Dr. Mark Rosker) and the FCRP IFC project of the Semiconductor Research Corporation.

\section{REFERENCES}

[1] Y. Cao, D. Deen, J. Simon, J. Bean, N. Su, J. Zhang, P. Fay, H. Xing, and D. Jena, "Ultrathin MBE-Grown AlN/GaN HEMTs with Record High Current Densities," Proc. Of the 2007 Int. Semiconductor Device Research Symp., College Park, MD, 2007.

[2] U. K. Mishra, L. Shen, T. E. Kazior, and Y.-F. Wu, "GaN-Based RF Power Devices and Amplifiers," Proc. of the IEEE 96, pp. 287-305, 2008 .

[3] J. W. Chung, O. Saadat, and T. Palacios, "Gate-recessed AlGaN/GaN HEMT with a Record fmax of $300 \mathrm{GHz}$," Proc. Of International Conference on Nitride Semiconductors, Jeju Island, South Korea, 18-23 October 2009.

[4] K. V. Smith, "GaN Reliability Through the Decade," Proc. Of the Meeting of the Materials Research Society, Boston, MA, 2008.

[5] Intel Press Release, "Intel Demonstrates Industry's First $32 \mathrm{~nm}$ Chip and Next-

GaN HEMTs," IEEE Electron Dev. Letts., in press, 2009.

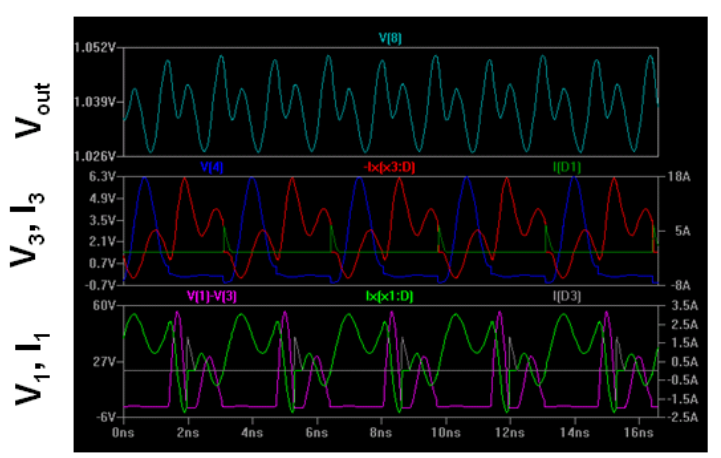

Fig. 6. Simulation of the current and voltage waveforms in the $12: 1 \mathrm{~V}$ hybrid voltage regulator studied in this project.

Generation Nehalem Microprocessor Architechture," Sept. 18, 2007.

[6] R. Fischer, T. Henderson, J. Klem, W. Kopp, C. K. Peng, and H. Morkoc, "Monolithic integration of $\mathrm{GaAs} / \mathrm{AlGaAs}$ modulation-doped field-effect transistors and N-metal-oxide-semiconductor silicon circuits," Appl. Phys. Lett., vol. 47, pp. 983-985, Nov. 1985.

[7] R. N. Ghosh, B. Griffing, and J. M. Ballantyne, "Monolithic integration of GaAs light-emitting diodes and Si metaloxide-semiconductor field-effect transistors," Appl. Phys. Lett., vol. 48, pp. 370-371, Feb. 1986.

[8] H. K. Choi, G. W. Turner, and B-Y. Tsaur, "Monolithic integration of $\mathrm{Si}$ MOSFET's and GaAs MESFET's," IEEE Electron Device Lett., vol. 7, no. 4, pp. 241-243, Apr. 1986.

[9] S. Joblot, F. Semond, Y. Cordier, P. Lorenzini, and J. Massies, "High-electronmobility $\mathrm{AlGaN} / \mathrm{GaN}$ heterostructures grown on $\mathrm{Si}(001)$ by molecular-beam epitaxy," Appl. Phys. Lett., vol. 87, 133505 , Sep. 2005.

[10]F. Schulze, O. Kisel, A. Dadgar, A. Krtschil, J. Blasing, M. Kunze, I. Daumiller, T. Hempel, A. Diez, R. Clos, J. Christen, and A. Krost, "Crystallographic and electric properties of MOVPE-grown AlGaN/GaN-based FETs on Si(001) substrates," J. Crystal Growth, vol. 299, pp. 399-403, Feb. 2007.

[11] J.W. Chung, J.-K. Lee, E. L. Piner, and T. Palacios, "Seamless On-Wafer Integration of Si (100) MOSFETs and 